\title{
Effect of Gallic Acid and Endurance Exercise Training on BDNF in a Model of Hippocampal Degeneration
}

\author{
Samaneh Rafiei ${ }^{1}$, Yunes Bazyar ${ }^{1}$, Mohammad Amin Edalatmanesh ${ }^{2 *}$ \\ ${ }^{1}$ Department of Exercise Physiology, Marvdasht Branch, Islamic Azad University, Marvdasht, Iran \\ ${ }^{2}$ Department of Physiology, College of Sciences, Shiraz Branch, Islamic Azad University, Shiraz, Iran
}

\section{A BSTRACT}

Introduction: The aim of this study was to investigate the effect of gallic acid on hippocampal level of brain derivative neurotrophic factor (BDNF) in trimethyltin chloride (TMT)- intoxication rats after eight weeks of endurance training. Materials and Methods: Seventy Sprague-dawley male rats were selected and randomly divided into seven groups, including: (1) Control, (2) Sham, (3) Gallic acid 50, (4) Gallic acid 100, (5) Endurance training (4), (6) Training + Gallic acid 50, and (7) Training + Gallic acid 100. Hippocampal degeneration was induced by TMT $(8 \mathrm{mg} / \mathrm{kg})$ in all groups except control animals. During eight weeks, rats of groups 3, 6 and 7 ran on treadmill's without incline at a speed of 15 to 20 meters per minute for 15 to 30 minutes per session, five times weekly. In addition, groups 4 and 6 were treated with gallic acid $(50 \mathrm{mg} / \mathrm{kg})$ and groups 5 and 7 with gallic acid $(100 \mathrm{mg} / \mathrm{kg})$ every day for 14 days. Results: The findings showed that hippocampal levels of BDNF in all test groups was significantly higher than sham group. Conclusion: Endurance training and gallic acid as well as the combination of them increase the level of hippocampal BDNF in a model of TMT-intoxication.

\section{Key words:}

1. Hippocampus

2. Physical Endurance

3. Gallic Acid

4. Brain-Derived Neurotrophic Factor

*Corresponding Author: Mohammad Amin Edalatmanesh

E-mail:amin.edalatmanesh@gmail.com 


\title{
اثر اسيدكاليك و تمرين ورزشى استقامتى بر فاكتور نوروتروفيك مشتق از مغز در يك مدل از دزنر اسيون هيبيو كامب بوري
}

\author{
سمانه رفيعى'، يونس بازيار '، محمد امين عدالت منش ז" \\ اكروه فيزيولوزى ورزشى، واحد مرودشت، دانشكاه آزاد اسلامى، مرودشت، ايران

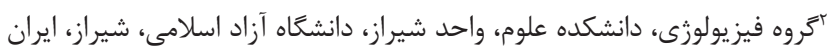

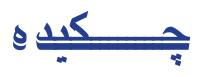

مقدمه: هدف از اين يزوهش، بررسى اثر اسيد اليك بر سطح هييو كاميى فاكتور نوروتروفيك مشتق از از

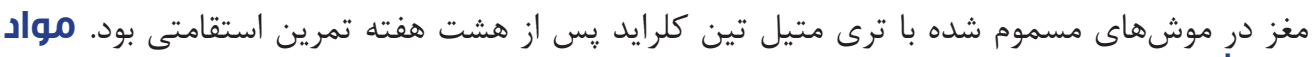

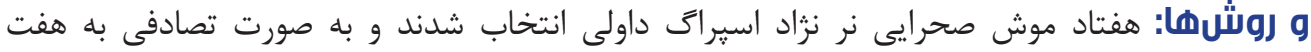

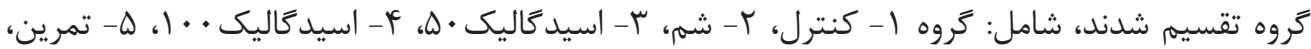

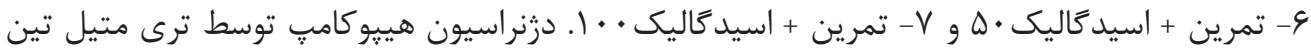

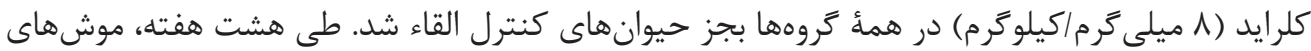

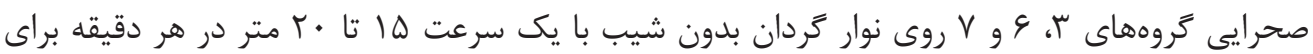

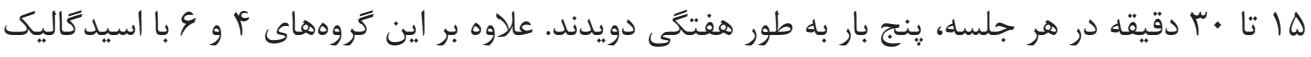

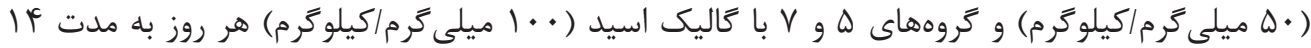

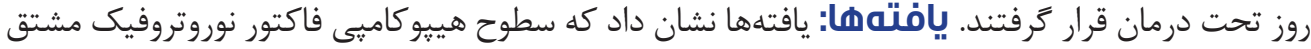

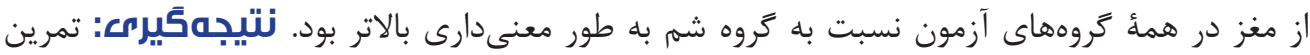

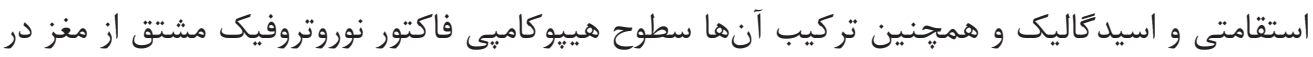

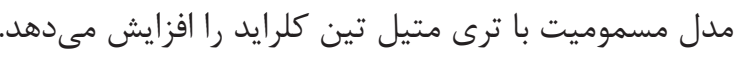

كليد وازهها:

ا

r. استقامت فيزيكى

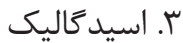

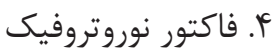

مشتق از مغز

* نويسنده مسئول: محمدامين عدالت منش : آدرس الكترونيكى: amin.edalatmanesh@gmail.com 


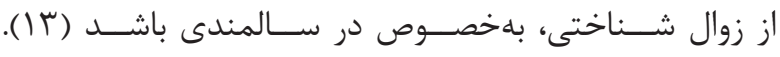

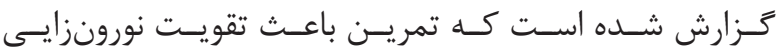

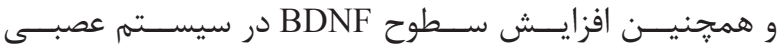

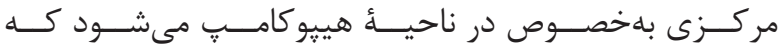

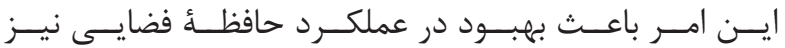

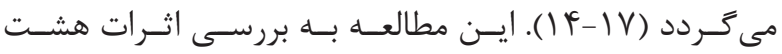

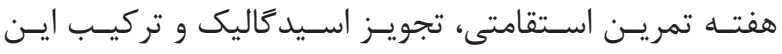

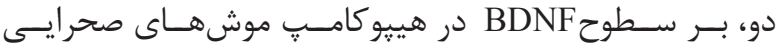

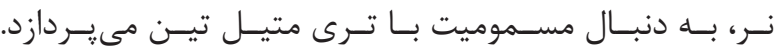

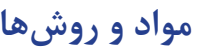

$$
\text { حيوانات و زروهبندى }
$$

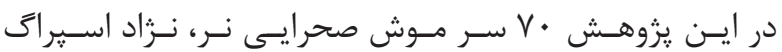

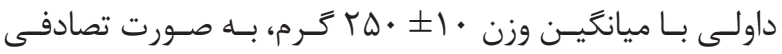

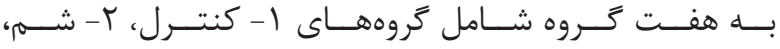

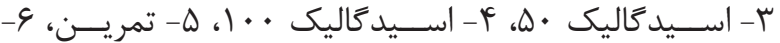

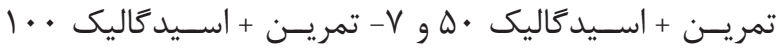

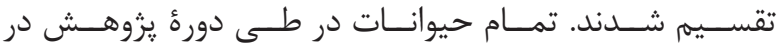

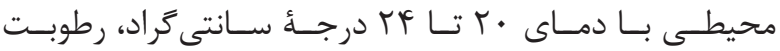
I T:I T Y

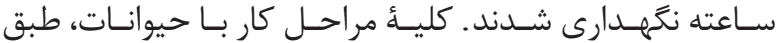

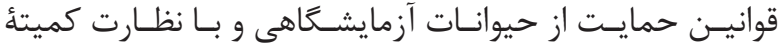

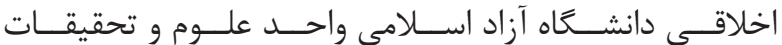

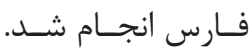

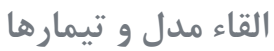

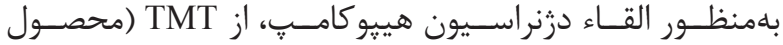

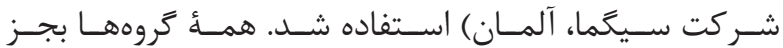

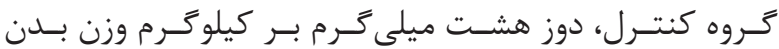

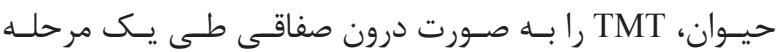

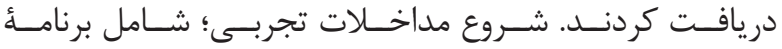

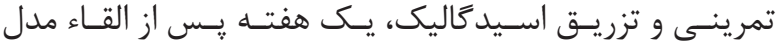

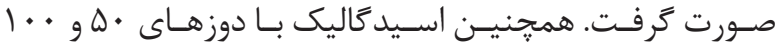

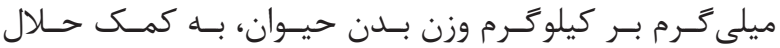

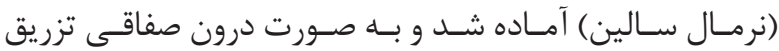

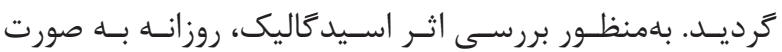

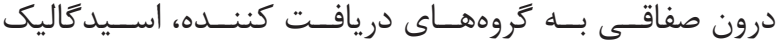

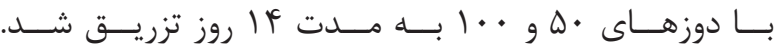

$$
\text { بروتكل تمرين }
$$

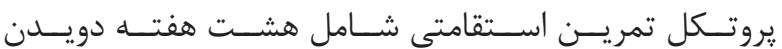

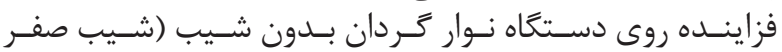

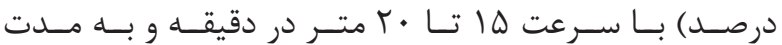

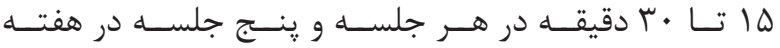

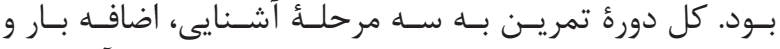

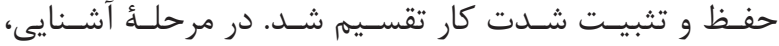

${ }^{1}$ Trimethyltin chloride

${ }^{2}$ Brain-derived neurotrophic factor

${ }^{3}$ Neurotrophin-3
مقدمه

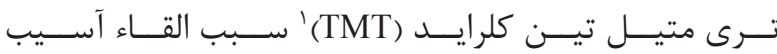

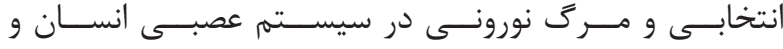

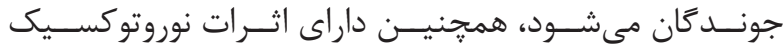

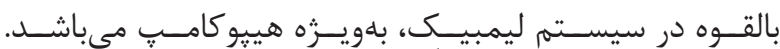

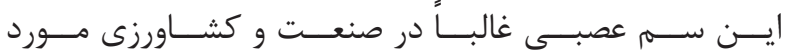

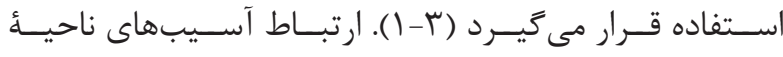

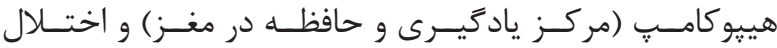

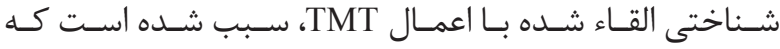

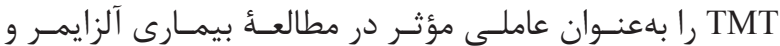

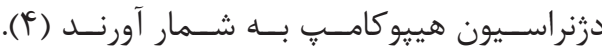

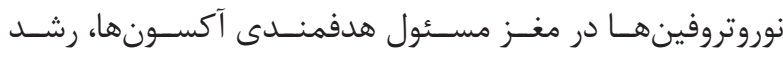

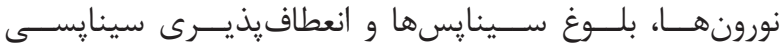

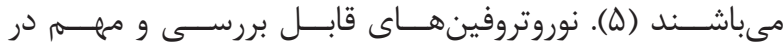

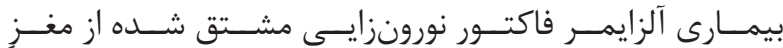

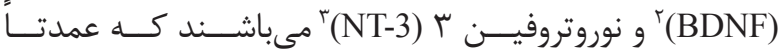

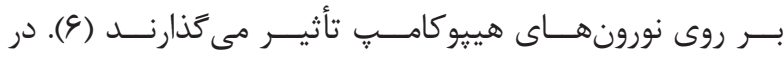

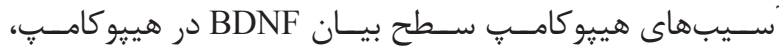

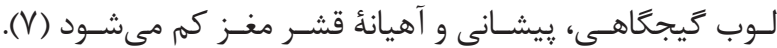

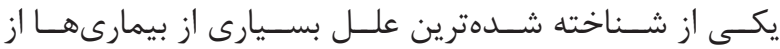

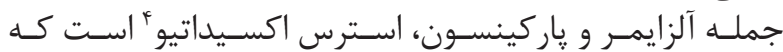

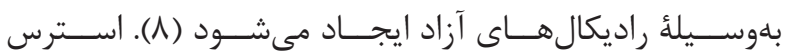

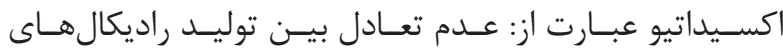

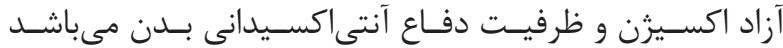

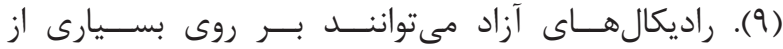

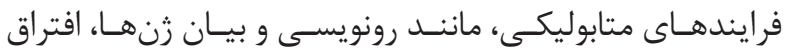

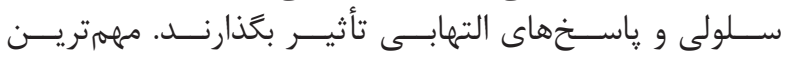

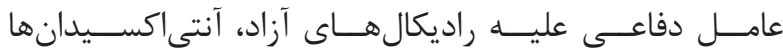

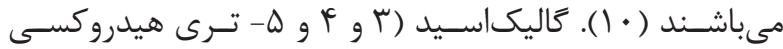

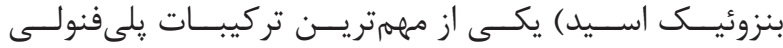

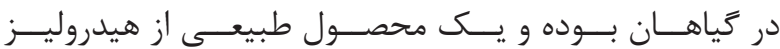

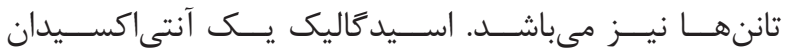

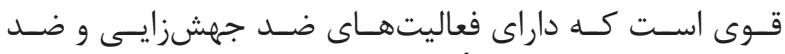

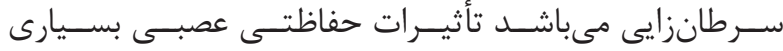

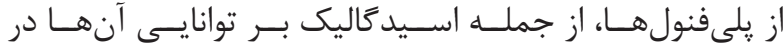

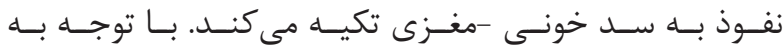

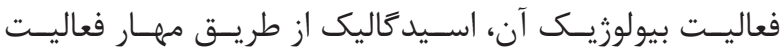

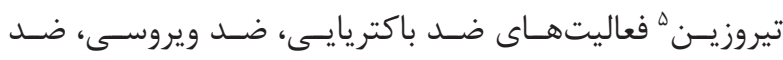

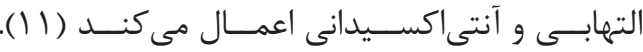

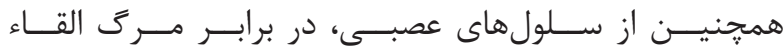

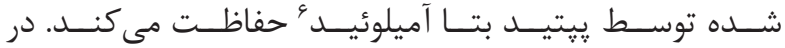

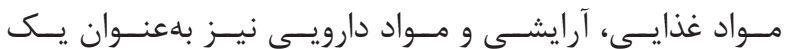

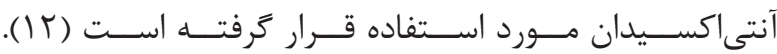

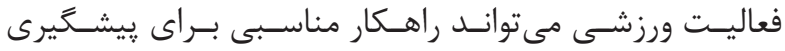

${ }^{4}$ Oxidative stress

${ }^{5}$ Tyrosine

${ }^{6}$ Amyloid beta-peptide 


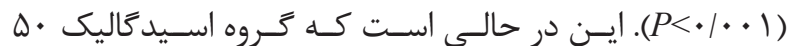

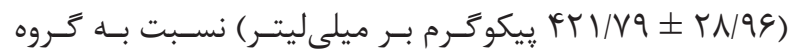

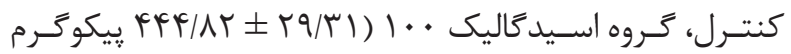

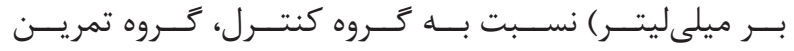

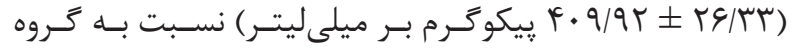

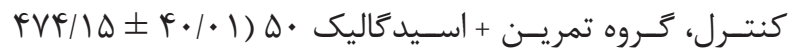

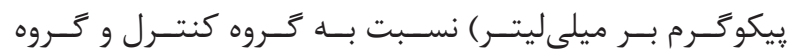

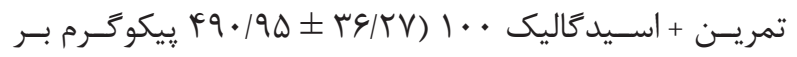

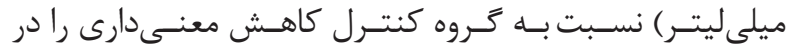

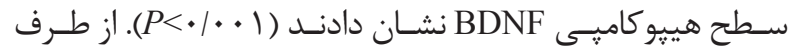

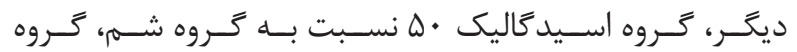

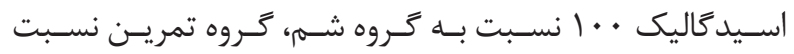

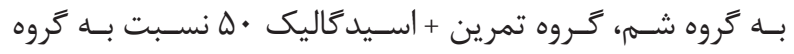

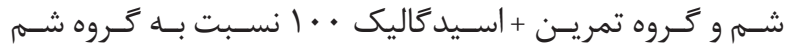

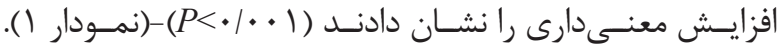

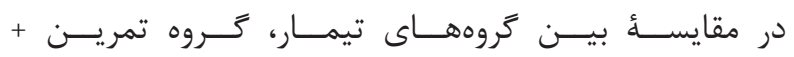

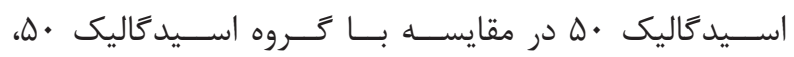

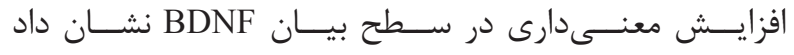

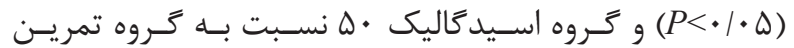

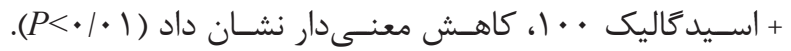

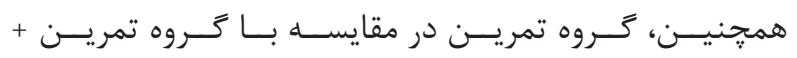

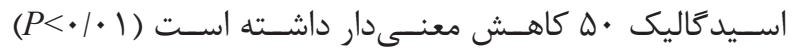

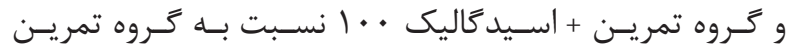

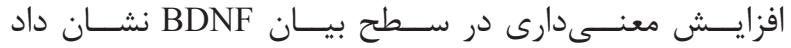

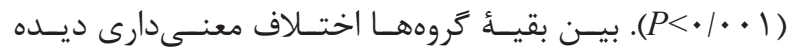

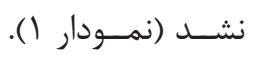

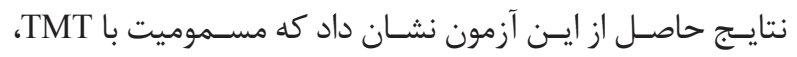

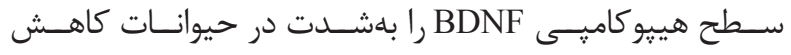

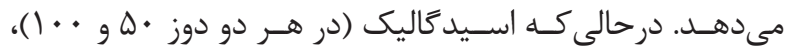

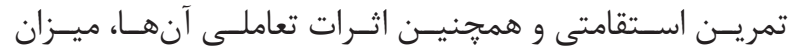

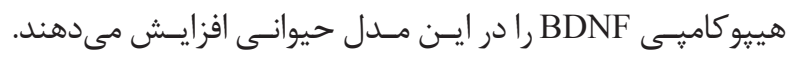

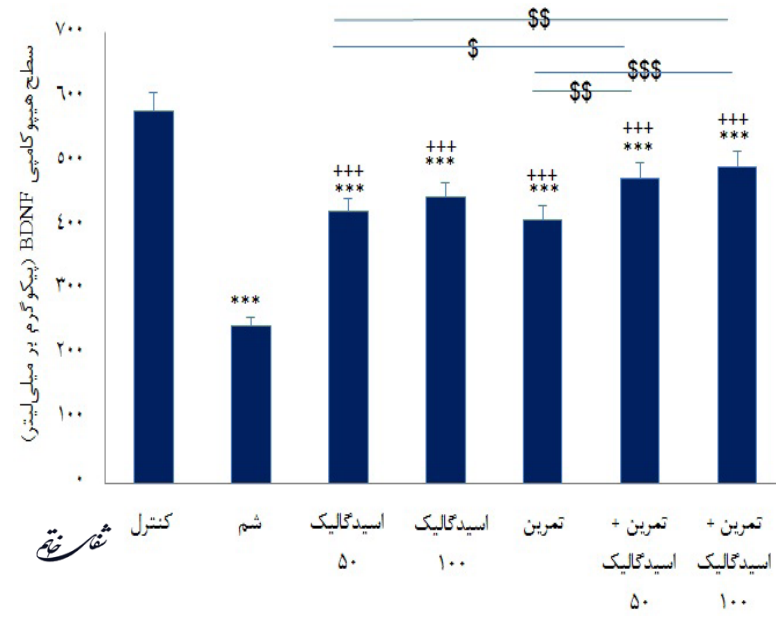

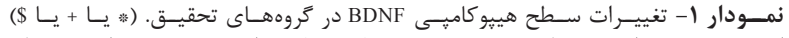

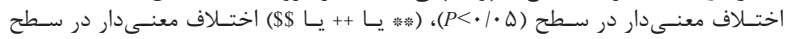
( )

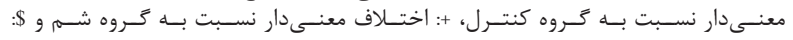

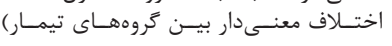

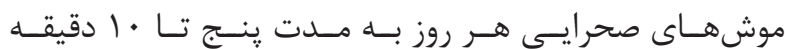

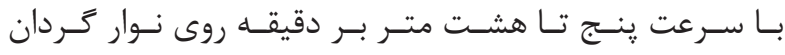

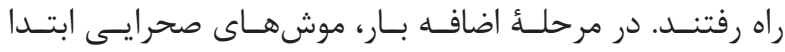

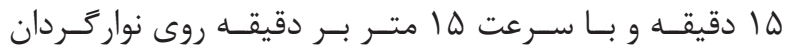

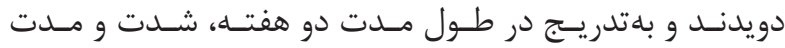

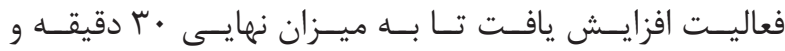

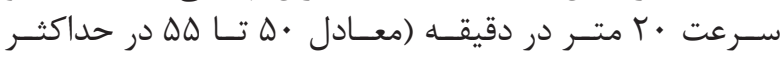

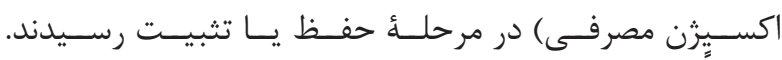

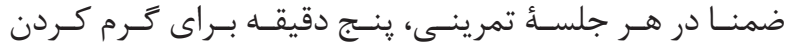

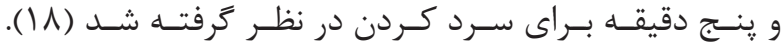

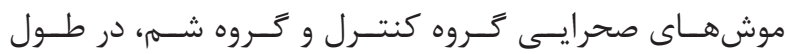

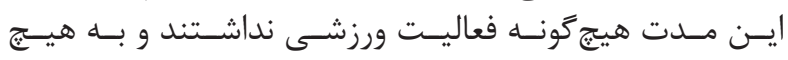

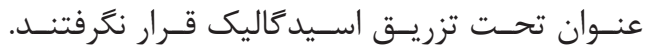

$$
\text { سنجش ميزان هيبوكاميى BDNF }
$$

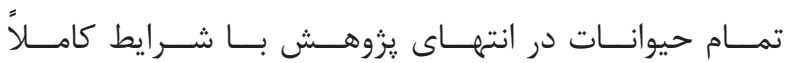

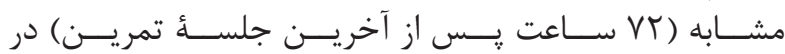

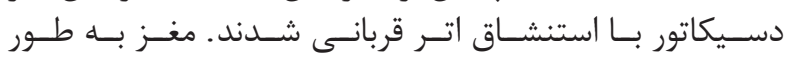

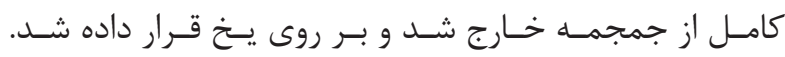

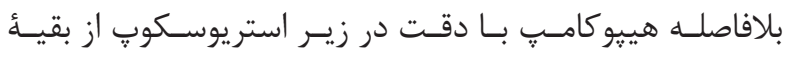

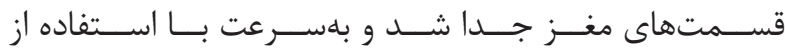

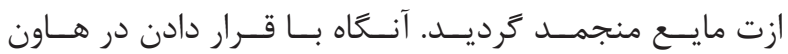

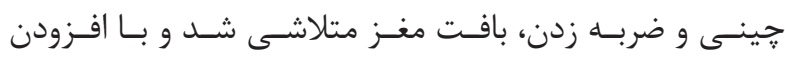

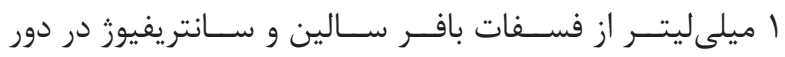

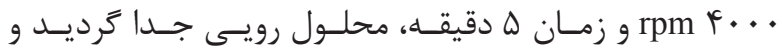

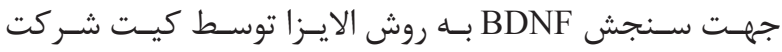

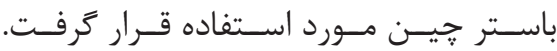

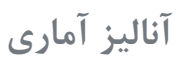

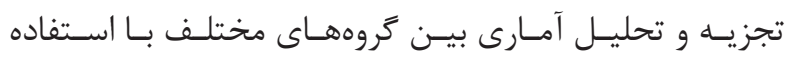

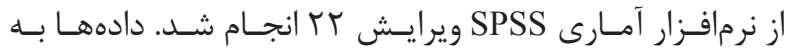

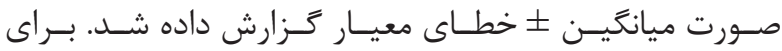

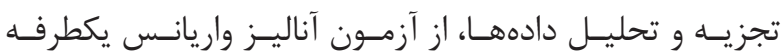

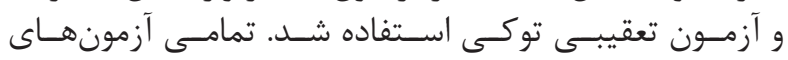

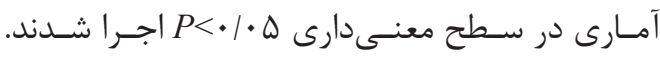

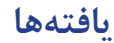

بررسى ميزان بيان هييوكاميى BDNF

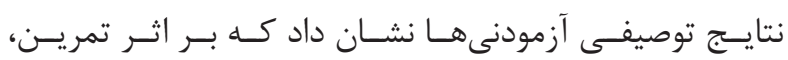

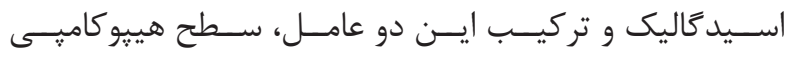

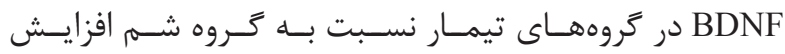

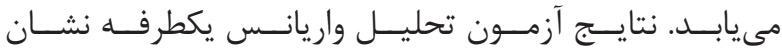

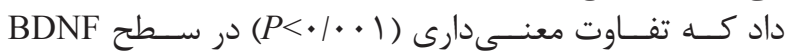

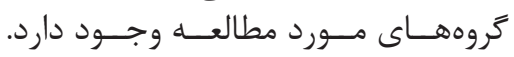

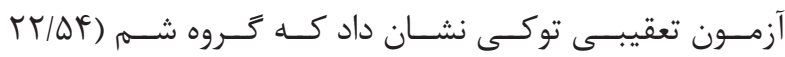

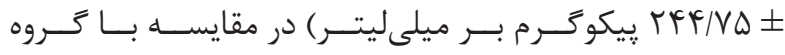

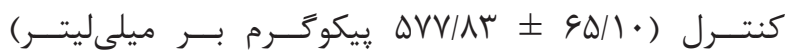

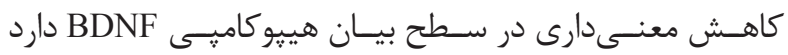




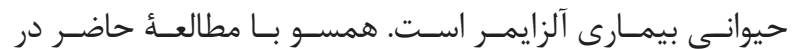

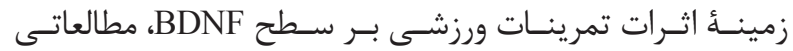

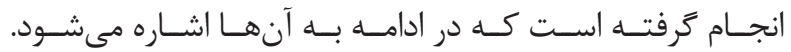
Cechetti

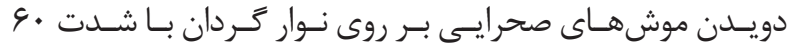

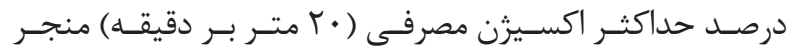

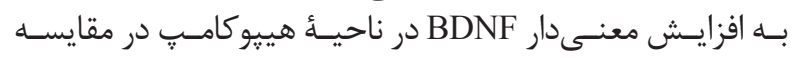

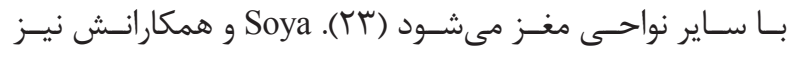

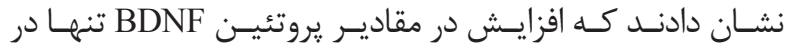

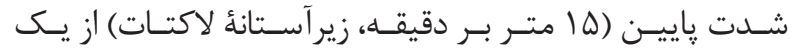

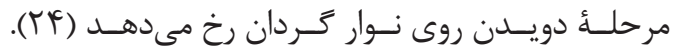

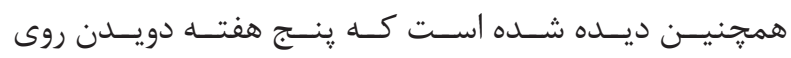

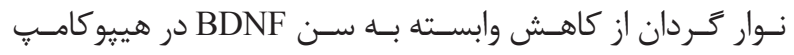

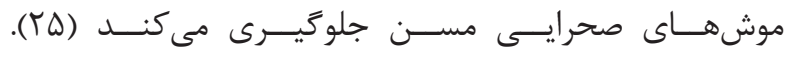

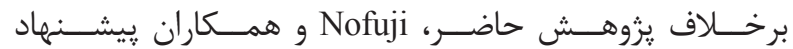

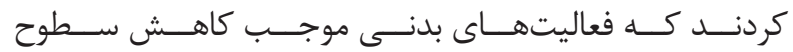
BDNF

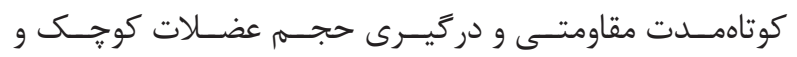

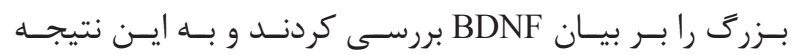

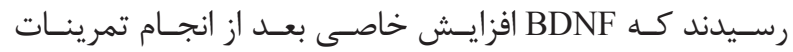

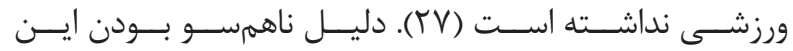

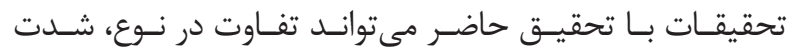

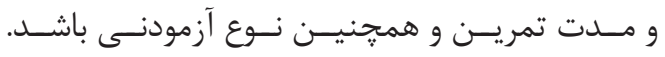

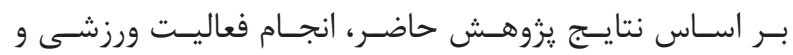

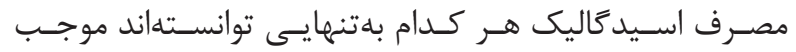

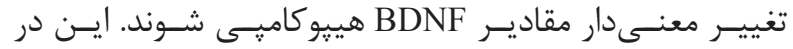

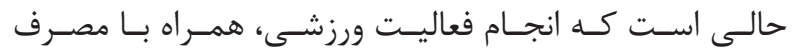

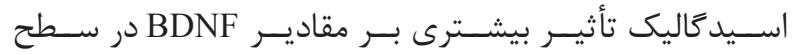

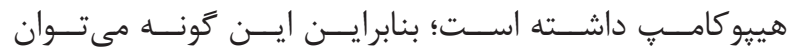

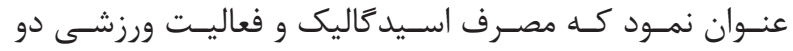

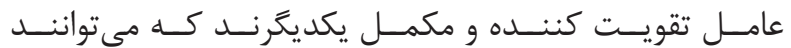

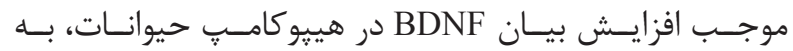

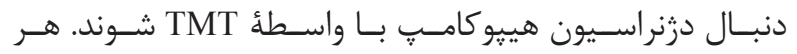

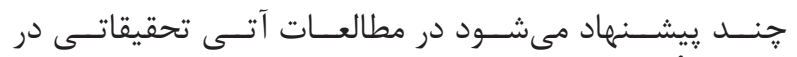

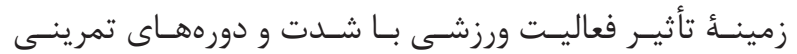

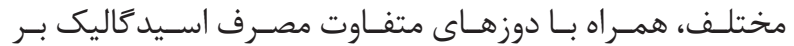

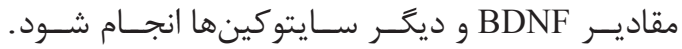

1. Pasinetti GM, Ho L. Role of grap seed polyphenols in Alzheimer's discase neuropathology. Nutr Diet suppl. 2010; 2: 97-103.

${ }^{7}$ Protocatechuic acid

${ }^{8}$ Tyrosol

${ }^{9}$ Caftaric acid

${ }^{10}$ Scutellarin

${ }^{11}$ Diadzein
بحث و نتيجه

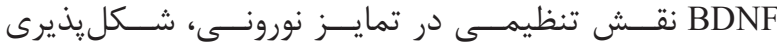

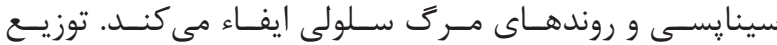
BDNF

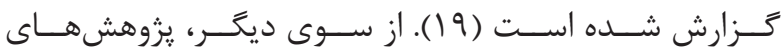

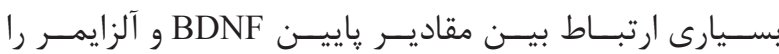

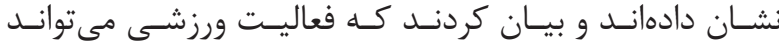

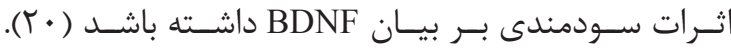

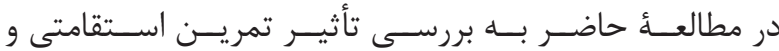

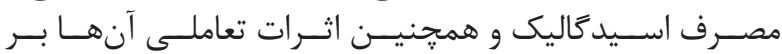

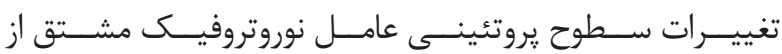

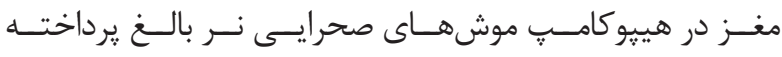

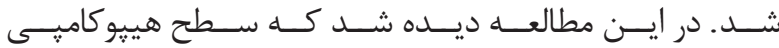
BDNF

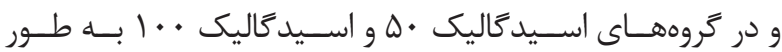

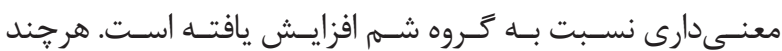

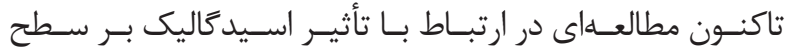

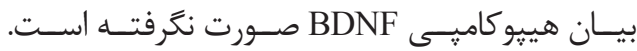

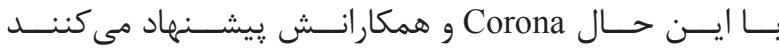

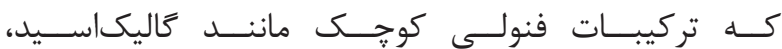

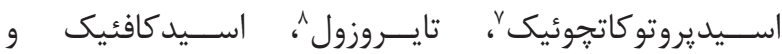

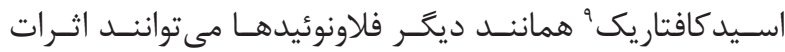

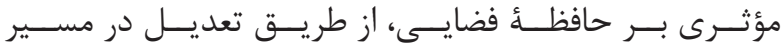

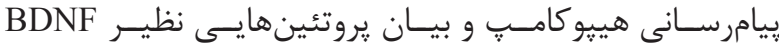

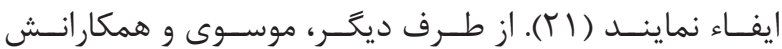

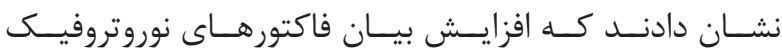

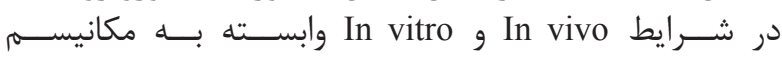

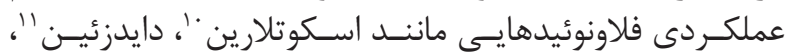

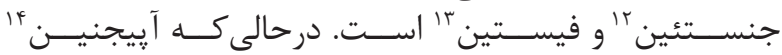

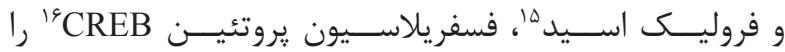

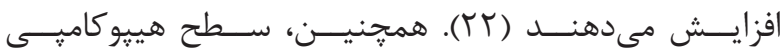

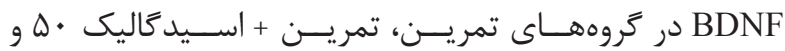

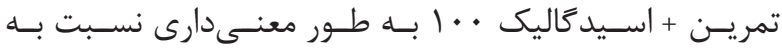

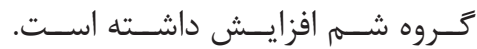

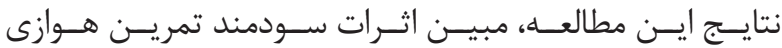

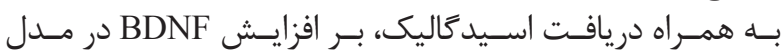

منابع

2.BesserJM,BrumbaughWG,BrunsonEL,IngersollCG.Acute and chronic toxicity of lead in water and diet to the amphipod Hyalella azteca. Environ Toxicol Chem. 2005; 24: 1807-15.

\footnotetext{
${ }^{12}$ Genistein

${ }^{13}$ Fisetin

${ }^{14}$ Apigenin

${ }^{15}$ Ferulic acid

${ }^{16}$ Cyclic adenosine monophosphate response element binding protein
} 
3. White L, Petrovitch H, Ross GW, Masaki KH, Abbott $\mathrm{RD}$, Teng EL, et al. Prevalence of dementia in older Japanese-American men in Hawaii: the Honolulu-Asia aging atudy. JAMA. 1996; 276(12): 955-60.

4. Corvino V, Marchese E, Michetti F, Geloso MC. Neuroprotective strantegics in hippocampal neuro degeneration induced by the neurotoxicant trimethylyin. Neurochem Res. 2013; 38(2): 240-53.

5. Autry AE, Monteggia LM. Brain-derived neurotrophic factor and neuropsychiatric disorders. Pharmacol Rev. 2012; 64(2): 238-58.

6. Castren E. Is mood chemistry? Nat Rev Neurosci. 2005; 6(3): 241-6.

7. Puglielli L. Aging of the brain, neurotrophin signaling, and Alzheimer's disease: is IGF1-R the common culprit? Neurobiol Aging. 2008; 29(6): 795-811.

8. Asghari N, Aliakbari M, Dadkhah A. The effects of group logotherapy on decreasing the degree of depression in female olds. MEJDS. 2012; 2(1): 31-8.

9. Mohajeri D, Doostar Y. Antioxidant effect of extract of the grape seed in streptozotocin induced diabetic rats. ZJRMS. 2010; 12(1): 9-14.

10. Koksal E, Gulcin I. Antioxidant activity of cauliflower. Turk J Agric. 2008; 32: 65-78.

11. Farbood Y, Sarkaki A, Hashemi Sh. The effects of gallic acid on pain and memory following transient global ischemia/reperfusion in wistar rats. Avicenna J Phytomed. 2013; 3(4): 329-40.

12. Mansouri MT, Farbood Y, Jafar Sameri M, Sarkaki A, Naghizadeh B, Rafeirad M. Neuroprotective effects of oral Gallic Acid against oxidative stress induced by 6hydroxydopamine in rats. Food chem. 2013; 138(2): 1028-33.

13. Vaynman S, Ying Z, Gomez PF. Hippocampal BDNF mediates the efficacy of exercise on synaptic plasticity and cognition. Eur J Neurosci. 2004; 20(10): 2580-90.

14. Gold SM, Schulz KH, Hartmann S, Mladek M, Lang UE, Hellweg R, et al. Basal serum levels and reactivity of nerve growth factor and brain-derived neurotrophic factor to standardized acute exercise in multiple sclerosis and controls. J Neuroimmunol. 2003; 138(1-2): 99-105.

15. Cotman CW, Berchtold NC. Exercise: a behavioral intervention to enhance brain health and plasticity. Trends Neurosci. 2002; 25 (6): 295-301.

16. Adlard PA, Perreau VM, Engesser-Cesar C, Cotman $\mathrm{CW}$. The time course of induction of brain-derived neurotrophic factor mRNA and protein in the rat hippocampus following voluntary exercise. Neurosci Lett. 2004; 363 (1): 43-8.

17. Seifert T, Brassard P, Wissenberg M, Rasmussen P, Nordby P, Stallknecht B, et al. Endurance training enhances BDNF release from the human brain. Am J Physiol Regul Integr Comp Physiol. 2010; 298(2): 372-7.

18. Ooyama K, Wu J, Nosaka N, Aoyama T, Kasai M. Combined intervention of medium-chain triacylglycerol diet and exercise reduces body fat mass and enhances energy expenditure in rats. J Nutr Sci Vitaminol (Tokyo). 2008; 54(2):136-41.

19. Kuipers SD, Bramham CR. Brain-derived neurotrophic factor mechanisms and function in adult synaptic plasticity: new insights and implications for therapy. Curr opin drug discov develop. 2006; 9(5): 580-6.

20. Navarro A, Sanchez DP, Gomez C, Peralta JL, Boveris A. Behavioral dysfunction brain oxidative stress and impaired mitochondrial electron transfer in aging mice. Am J PhysiolRegulIntegr Comp Physiol. 2002; 282: 985-92.

21. Corona G, Vauzour D, Hercelin J, Williams CM, Spencer JP. Phenolic acid intake, delivered via moderate champagne wine consumption, improves spatial working memory via the modulation of hippocampal and cortical protein expression/ activation. Antioxid Redox Signal. 2013; 19(14): 1676-89.

22. Moosavi F, Hosseini R, Saso L, Firuzi O. Modulation of neurotrophic signaling pathways by polyphenols. Drug Des Devel Ther. 2015; 10: 23-42.

23. Cechetti F, Fochesatto C, Scopel D, Nardin P, Gonçalves CA, Netto CA. Effect of a neuroprotective exercise protocol on oxidative state and BDNF levels in the rat hippocampus. Brain Res. 2008; 1188: 182-8.

24. Soya H, Nakamura T, Deocaris CC, Kimoara A, Iimura M, Fujikawa T. BDNF induction with mild exercise in the rat hippocampus. Biochem Biophys Res Communi. 2007; 358(4): 961-7.

25. Wu A, Ying Z, Gomez PF. Docosahexaenoic acid dietary supplementation enhances the effects of exercise on synaptic plasticity and cognition. Neuroscience. 2008; 155(3): 751-9.

26. Nofuji Y, Suwa M, Moriyama Y, Nakano H, Ichimiya A, Nishichi R, et al. Decreased serum-brain derived neurotrophic factor in trained men. Neurosci Lett. 2008; 437(1): 29-32.

27. Corria PR, Pansani A, Machado F, Andrade M, da Silva AC, Fulvio Alexandre, Scorza FA, et al. Acute strength exercise and the involvement of small or large muscle mass on plasma brain-derived neurotrophic factor levels. 2010; 65(11): 1123-6. 